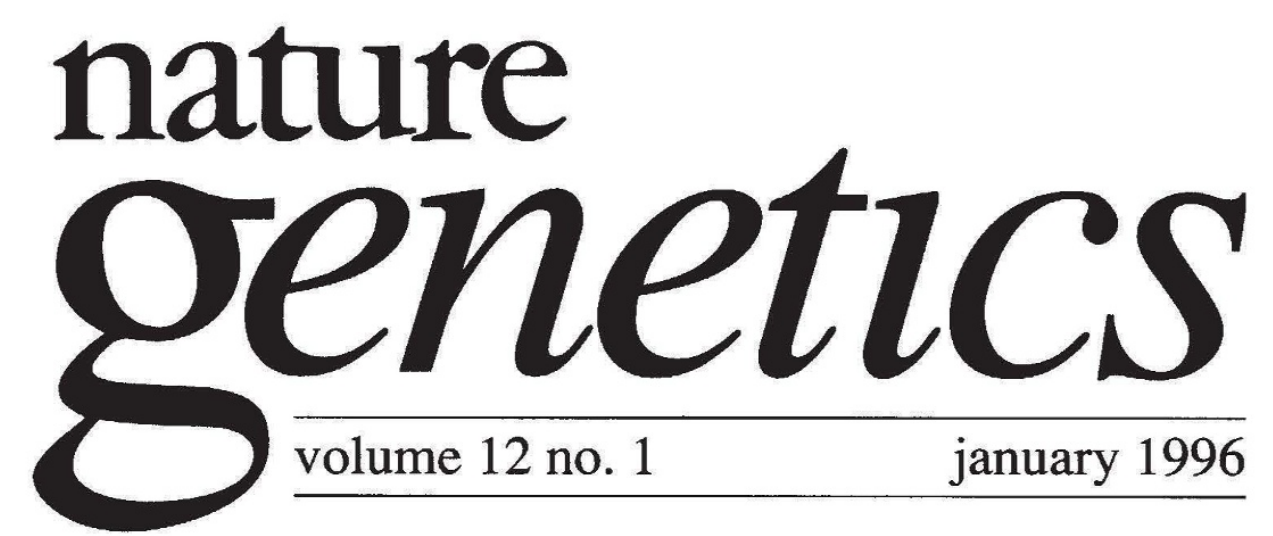

\title{
Third and long (QT)
}

It has been an exciting year for the cardiology field, especially in view of the remarkable insights emerging into one of the better known heart disorders, the long-QT syndrome (LQT). This genetically heterogenous disease is a rare but quite well known form of sudden death, resulting from a defect in cardiac repolarization that leads to ventricular arrhythmia ${ }^{1}$. Never far from the public eye, the tragedy of sudden heart failure struck again recently with the collapse and death of Sergei Grinkov, the Russian ice-skating star, who was just 28 years old. Grinkov was found to have had severe heart disease (his father had died from similar causes in his mid-fifties) that was unrelated to strictly hereditary causes such as LQT or familial hypertrophic cardiomyopathy (FHC). But there is no doubt that uncovering the molecular basis of these hereditary forms of heart disease will go a long way towards improving our understanding of cardiac physiology and treating the problem.

Six months ago, Mark Keating and his colleagues at the University of Utah in Salt Lake City reported that they had identified a pair of genes responsible for two forms of the autosomal dominant (Romano-Ward) form of LQT, using a candidate gene approach ${ }^{2,3}$. The first, HERG, is situated on chromosome $7 \mathrm{q} 35-36$ and encodes a potassium channel ${ }^{2}$ related to the delightfully named ether-a-gogo gene from Drosophila. The second, SCN5A, codes for a sodium channel ${ }^{3}$ and maps to chromosome 3p21-24. These two loci, known as LQT2 and LQT3 respec-

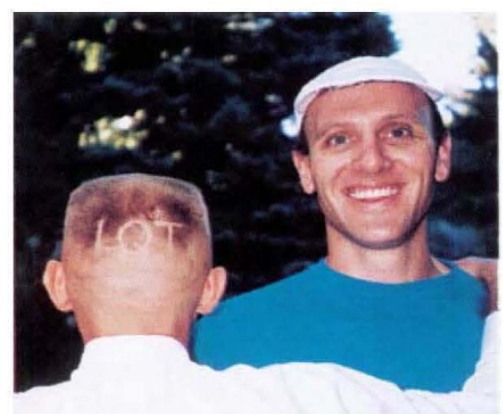
tively, revealed much about the mechanisms of the disease, but the cause of the most common form of the disorder (50-60 per cent of cases), due to defects in $L Q T 1$, has been a mystery since the Utah group first mapped the gene to the short arm of chromosome 11 in 1991. As described on page 17 of this issue, Keating's group has started off 1996 in grand style by presenting the gene for LQT1. Interestingly, the gene turns out to be another potassium channel that is also most likely involved in cardiac repolarization ${ }^{4}$.

The initial localization of $L Q T 1$ a few years ago had raised the tantalizing possibility that the gene in question might be the RAS oncogene that resides in the vicinity (RAS is known to regulate cardiac potassium channels), but subsequent studies excluded the oncogene. Since then, the loci for three other forms of the disease (most recently LQT4 on chromosome 4q25-27) have been mapped, and two of the genes themselves isolated. Not surprisingly, the Utah team hoped that their identification of specific potassium and sodium channel defects in the first two forms of LQT would simplify the tedious task of isolating the LQT1 locus by 

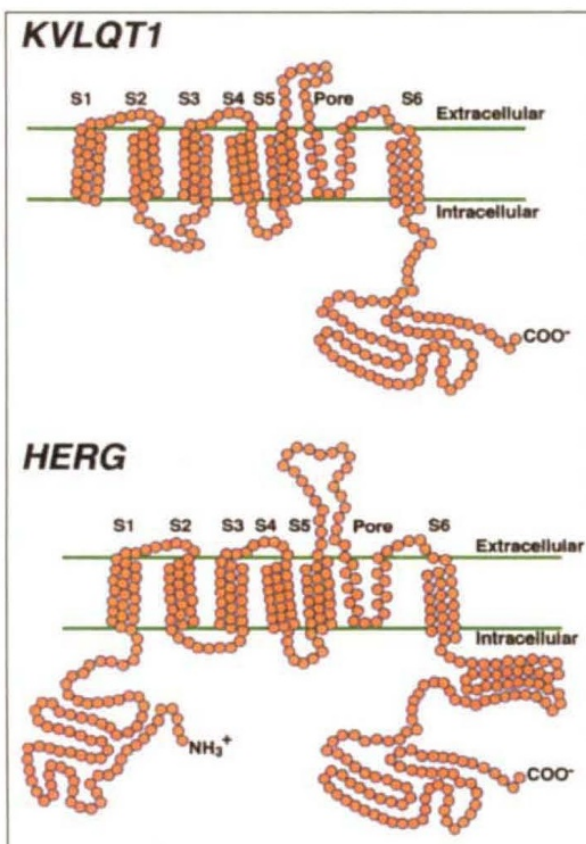

\section{SCN5A}

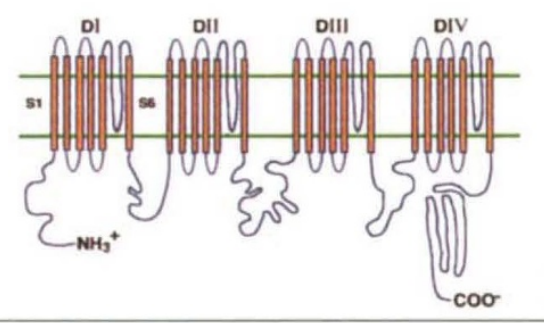

And then there were three: Topography of the three cardiac ion channels defective in long-QT syndrome: potassium channels KVLQT1 and HERG, and the sodium channel SCN5A. positional cloning. But although they mapped two potassium channel genes to the critical region, neither proved to be the gene they were after.

Wang et al. ${ }^{4}$ consequently stuck to the tried and trusted technique of exon amplification, which yielded eight short fragments perhaps representing portions of ion-channel genes. The full cDNA encompassing all eight of these exons did indeed encode a potassium channel gene with six putative membrane-spanning regions and $30 \%$ identity to the shaker channel. In the largest available family with the disorder (K1532), with 70 affected members, Wang et al. detected a valine-to-methionine missense mutation at codon 125 in the intracellular loop between the fourth and fifth membrane-spanning regions. Mutations have so far been identified in 16 families, all but one of them missense mutations; interestingly, seven of the 15 result in substitutions of alanine 212 in the sixth transmembrane domain.

The precise functional role of the newly cloned potassium channel, KVLQT1, remains to be determined, but the indications are that, like HERG, it acts as a voltage-gated channel involved in cardiac repolarization. (Indeed, it is possible that it forms heterotetramers with HERG.) Wang et al. suggest that the mutations may act in a dominant-negative capacity, causing the tetrameric potassium channels to lose function. The Utah group has already expressed HERG, the other potassium channel associated with the disease, in Xenopus oocytes. Those experiments demonstrated that HERG exhibits properties very similar to the outwardly rectifying potassium channel found in cardiac myocytes ${ }^{5}$. In new work, Keating's group has resolved an earlier contradiction and found that HERG can be blocked under certain conditions by methanesulfonanilides, known blockers of the cardiac myocyte potassium current ${ }^{6}$. Moreover, they have shown that LQT mutations in the HERG channel act in a dominant-negative fashion ${ }^{7}$.

The effect of an LQT-associated mutation in the sodium channel SCN5A has also been investigated in an oocyte expression system. Bennett et al. ${ }^{8}$ found that mutant channels containing a three-amino-acid deletion (residues 1505-1507), as documented in two affected families, show a prolonged inward current due to the tendency of some channels to switch to a non-inactivating mode, as observed by single channel recording. The result of the sustained inward current is a delay in repolarization of the cardiac action potential and an increased Q-T interval.

The latest findings on LQT offer further evidence for a unifying trend in the molecular basis of the disease - a trend that, as noted ${ }^{9}$ recently by Jeffrey Towbin (a co-author of the new KVLQT1 paper), resembles that described for the familial hypertrophic cardiomyopathies (FHC). As reported in last month's Nature Genet$i c s^{10,11}$, a new locus, that for myosin binding protein- $C$, has been added to the three previously identified genes ( $\beta$-cardiac myosin heavy chain, a tropomyosin and cardiac troponin $\mathrm{T}$ ) responsible for FHC, confirming the notion that the disease represents a 'disease of the sarcomere'.

With the majority of LQT cases now at least partially understood at the molecular level, there should be rapid progress in finding out how to treat these disorders. Early indications are that the sodium channel abnormality, which is caused by the inappropriate action of SCN5A, can be treated with sodium channel blockers that have proved successful in skeletal muscle disorders such as paramyotonia congenita. The LQT2 defect caused by mutations in HERG might be treatable by raising the concentration of potassium in the serum, which should activate the channel. Finally, the more common KVLQT1 defect should respond to drugs that open potassium channels. 